\title{
Information Quantity Model Applied in Hazard Evaluation of Landslides—A Case Study of Fuling, Chongqing
}

\author{
WenHao $\mathrm{Ou}^{1+}$, BinBin Zhao ${ }^{1}$, Yue Dai ${ }^{2}$ \\ ${ }^{1}$ Power transformer engineering research institute, China Electric Power Research Institute, Beijing \\ ${ }^{2}$ National Marine Hazard Mitigation Service, Beijing
}

\begin{abstract}
Based on RS, GIS technology and information quantity model, this paper will focus on the research of hazard evaluation method of landslides disaster in Fuling, Chongqing. Combining with theoretical basis of information quantity model, the evaluation method of this paper gives an improved model, and adds impacts of four dynamic factors: rainfall, water level changes, vegetation and landuse. It was agreed that the hazard evaluation result of landslides can be divided into five grade level: very high, high, moderate, low and very low. According to the comprehensive evaluation, the result shows that the improved information quantity model and the additional dynamic effects are reasonable, and demonstrates that this method is objective and can provide effective guarantee for geological disaster prevention of the research area. The whole evaluation functions of the hazard evaluation system of landslides are finally realized on the ArcGIS platform with its modelling capabilities and custom development mode.
\end{abstract}

Keywords: information quantity model, hazard evaluation, remote sensing, GIS.

\section{Introduction}

After the information quantity model was first used in the field of communications, it was introduced to the spatial prediction of geological disasters in 1989 because of its clear concept and the advantages of convenient calculation on GIS solutions. As more and more applications by the information quantity model, not only rationality has been confirmed, but also the model itself has been developed. It is mainly developed in three aspects: First, to increase the factor types of landslide model calculation, the second to build a variety of convenient forms of calculations based on the original model, the third is to explore suitable computing unit.

The information quantity model was first used in the field of communications, in 1989 and was introduced to the prediction of geological disasters in space, because of its clear concept, combining the advantages of convenient calculation GIS is widely used ${ }^{[1]}$. As more and more applications, not only rationality information model has been confirmed, the model itself has been developed. Development model is mainly reflected in three aspects: First, to increase the participation of landslide factors model calculation, the second is derived from a variety of convenient forms of calculations based on the model of the original formula, the third is to explore suitable computing unit.

This paper summarizes some variations of the information quantity model from applications, combining its theoretical basis, and analyses their strengths and weaknesses in aspects of the relevance of factors and weights. We study the impacts of calculation unit on the calculation, and gives their respective scope, which including point, surface and body unit. According to the restriction relations between internal factors of landslide, variations and calculation unit, an improved calculation process of information quantity model is proposed.

\footnotetext{
+ Corresponding author. Tel.: +8618611580926

E-mail address: owhnet@qq.com.
} 
The research adds rainfall, water level, land use and vegetation covered information four dynamics calculations complement to information quantity model, to some extent reducing the uncertainty, making landslides hazard assessment prediction results more accurately, and it has also been integrated into the hazard evaluation system of landslides automated process.

\section{Information Quantity Model}

\subsection{Expression}

The information quantity is a concept to reflect the certain probability of events occur in a combination of factors. Y represents disaster events, and $x_{i}(i=1,2 \ldots)$ represents the different causal factors, for different values xi which represents the different factors for the $i$ factor, as shown in Equation 2-1 [2].

$$
I\left(y, x_{1}, x_{2}, \ldots, x_{n}\right)=\log _{2} \frac{P\left(y \mid x_{1} x_{2} \cdots x_{n}\right)}{P(v)}
$$

$I\left(y_{3} x_{1}, x_{2}, \ldots, x_{n}\right)$ represents a combination of the information of factors $\left(x_{1}, x_{2}, \ldots, x_{n}\right)$, $P\left(y \mid x_{1} x_{2} \cdots x_{n}\right)$ represents a combination of the probability of occurrence under the disaster factors $\left(x_{1}, x_{2}, \ldots, x_{n}\right), P(y)$ indicates the normalized probability of occurrence of landslides. The result shows that meaningful information obtained by a combination of factors which represents a significant contribution to the landslide.

\subsection{Variation of calculating unit}

Since the information model is based on statistical probability model, it must relate to the concept of the sample and the totality. In landslide spatial prediction field, because of different scales, landslides can be shown by different symbols, so the sample unit contains types of point, surface and column. Also the form is diverse from these calculation unit of expression.

\subsubsection{Point in the form of the model}

Landslide points for characterizing the geographic location of landslides, usually a dot represents a specific landslide, in a relatively small scale map can be determined based on the coordinates of the location, with the increase of the scale, landslides can be identified in whole map, and then the landslide point can no longer complete express the specific circumstances of a landslide. The point expression in the form of landslide is represented with $N$ and $N_{i}$, which means the total number of landslides in the region and the number of landslides in the $x_{i}$ area factor, $A$ and $A_{i}$ represents the area of the total region area and factor $x_{i}$ respectively. The quantity of information through point information model can be written as the formula $2-2$ below [3].

$$
I_{i}=\log _{2} \frac{P\left(y \mid x_{i}\right)}{P(v)}=\log _{2} \frac{N_{i} / A_{\tilde{I}}}{N / A}=\log _{2} \frac{N_{i} / N}{A i / A}
$$

The advantage of point quantity information model is the convenience of calculation, but it should be considered that the point does can be represented the entire information of the landslide.

Point quantity information model is derived from the distribution of the point of landslide and the factors relations in space, but put aside their spatial relationship, we can do another explanation to the form of the model. The meaning of factors $x_{i}$ from $N_{i}$ expanses from region to interval, but also fully consistent with the principles of information quantity model, thus expanding the application range of the model.

\subsubsection{Surface in the form of the model}

Surface selection in middle scale of landslide is much more difficult for disaster bodies which influence by a wide-range scale, it is characterized by the scope of the surface and the level from overlap, but consider in a vertical direction, statistics of the surface on the point, is more complicated, mainly due to the shape and size which are not the regular surface. Equal to the quantity information model based on GIS technology supports an easy calculation method, which used to be more common used in raster layers. It shows the rule of the study area divided into square unit area, the landslide surface is also converted to a square unit, so we can get a statistical area by the number of grid statistics [4, 5]. The formula of grid computing the quantity information model in the single-factor shows as the formula 2-3. 


$$
I_{i}=\log _{2} \frac{P\left(y \mid x_{i}\right)}{P(v)}=\log _{2} \frac{S_{i} / A_{i}}{S / A}=\log _{2} \frac{N_{0} / W^{2}}{N_{n} / N}
$$

In the formula, $\mathrm{S}$ and $\mathrm{Si}$, respectively, represent the total surface area of the landslide and the landslide area in the factor of $x_{i}$ region, $N_{0}$ and $N_{0}^{i}$ represent the total number of landslides in raster grid and the specific area of landslide of factor $x_{i} . N$ and $N^{i}$, respectively show the total number of grids in the study area and the number of grid of factor $x_{i}$ area.

\subsubsection{Column in the form of the model}

The column forms of landslide is usually studying in the aspect of mechanical properties and motion mechanism by a single landslide. In general, the quantity of information model is rarely used as a landslide computing unit. The main reason that not to use the column of landslide was unnecessary, because the column of landslide appeared to calculate a large scale area, their specific circumstances often require a field survey, and regional landslide spatial hazard evaluation can be completed at the small scale, there is no need to consume a lot of manpower and resources. But some macroscopic properties of the landslide, such as length of slope, height of slope, direction of slope, etc., can be easily obtain from the landslide surface and topographic maps, we can use the information as a parameter of the quantity information model.

\section{Landslide Spatial Prediction Model}

\subsection{Current exist Problems}

In summary, the combination of factors, formula and calculation unit is depended on the restricting relationship between the information quantity models which do the geological hazard assessment, it mainly shows in the following aspects. Firstly, selection of the formula form is limited by the correlation of factors. Secondly, different factors need to select corresponding suitable computing units. Thirdly, the form of formula determines whether we can select a variety of computing units.

But in most studies currently, we often ignore one or several of the above constraints relationship, the common problems are two categories: One is selecting a single factor without any analysis of corresponding relationship between various factors, and the other one is select the same calculation unit for all factors. While these two issues are not necessarily have an impact on the results of the evaluation in some specific cases, but in the discussion of the usage of all types of cases must be classified before the calculation processes of information quantity model.

\subsection{ArcGIS model builder platform}

ESRI's ArcGIS software contains spatial model builder with many tools, which is a work flow method that enables the creation and execution of consistent, repeatable models that are comprised of one or more processing steps. Model builder can be used to ensure the integrity of a particular model or set of analytical processes through modelling, storing, and publishing complex operations and workflows.

Model builder workflows can be created and executed on both the desktop and over the web. Within model builder, a model must consists of processes and the connections between them. Parameters of the model process can be defined like which will be filled into a pop-up form at runtime. Model workflows also can be rerun with different data or inputs for evaluating scenarios. What's more, models created by the model builder workflow also can be exported as some scripts utility in Python and other programming languages, enhancing the Customization.

\subsection{The flow of expanded calculation}

Considering restricting relation of landslide internal factors, we get an improved information quantity model with the analysis of various modified form and calculating unit (Fig. 1). Information quantity under the combined effect of internal and external trigger factors has been calculated by adding rainfall, water level changes and vegetation to internal factors. The larger information quantity of this area describes that the combination of factors in the region is more conducive to breeding landslide.

In order to obtain a comprehensive hazard evaluation of the landslide, land use reflects the vulnerability of the affected object, which can help modify grade of the hazard evaluation. 


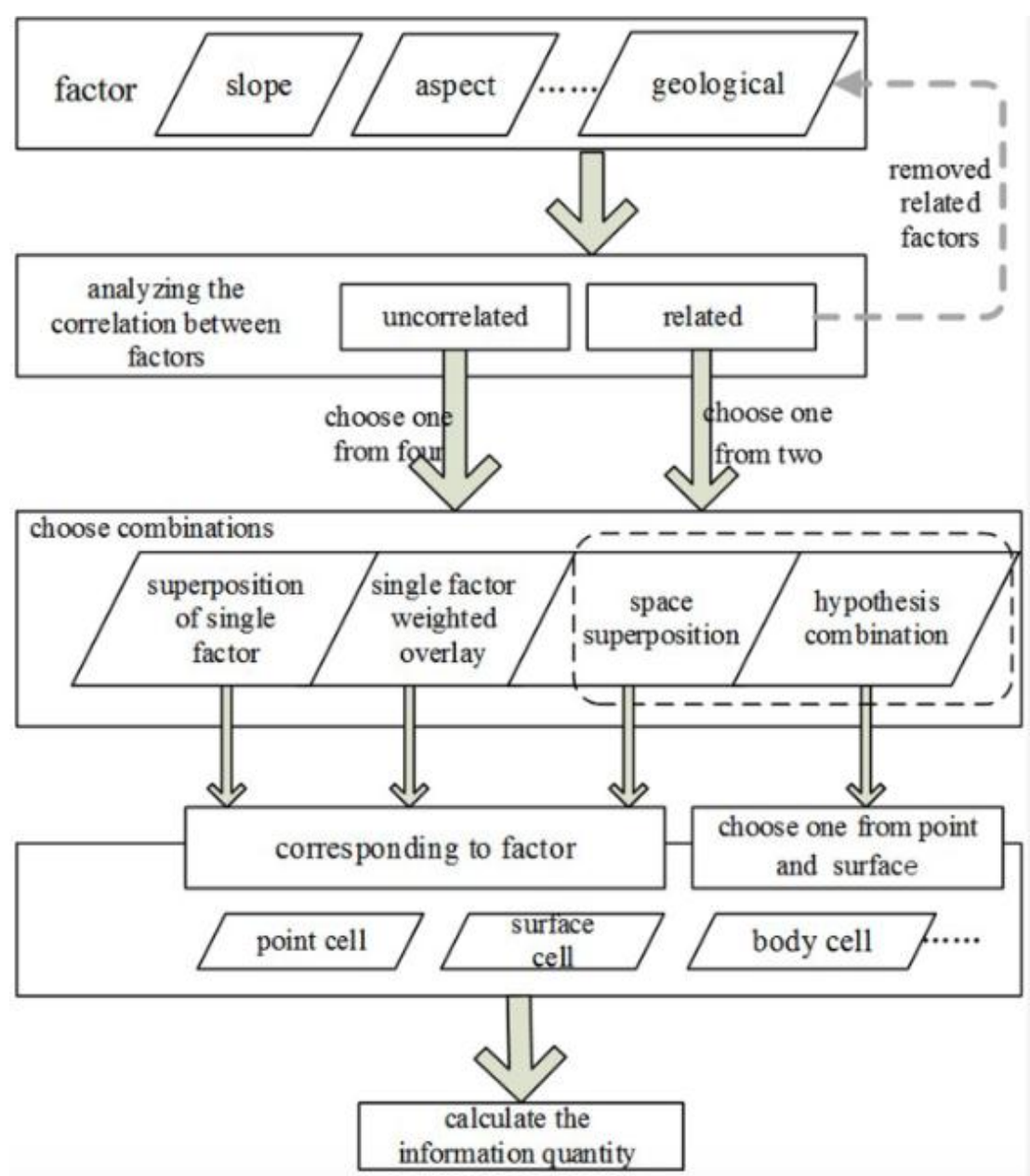

Fig. 1: The calculation flow chart of improved quantity information model.

We use ratio of disaster area and comparative validation to evaluate this method. The results verify the rationality of the improved information quantity and hazard evaluation method by considering external dynamic factors. Fig. 1 shows the workflow of improved information quantity model. Fig. 2 is the hazard evaluation model in system design. Fig. 3 shows the improved information quantity model applied in system in Fuling, Chongqing.

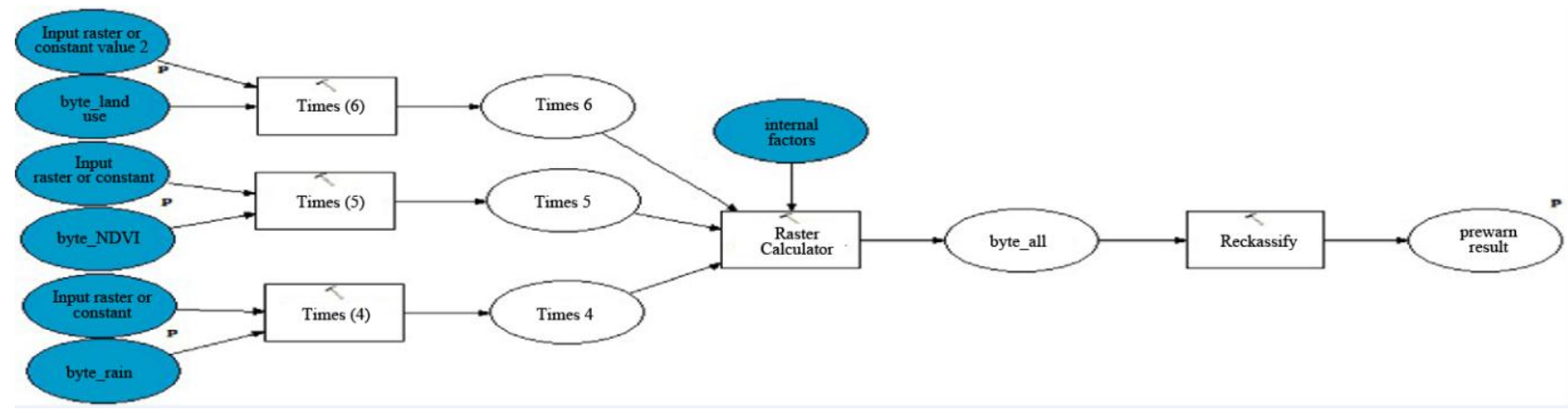

Fig. 2: Hazard evaluation method model. 


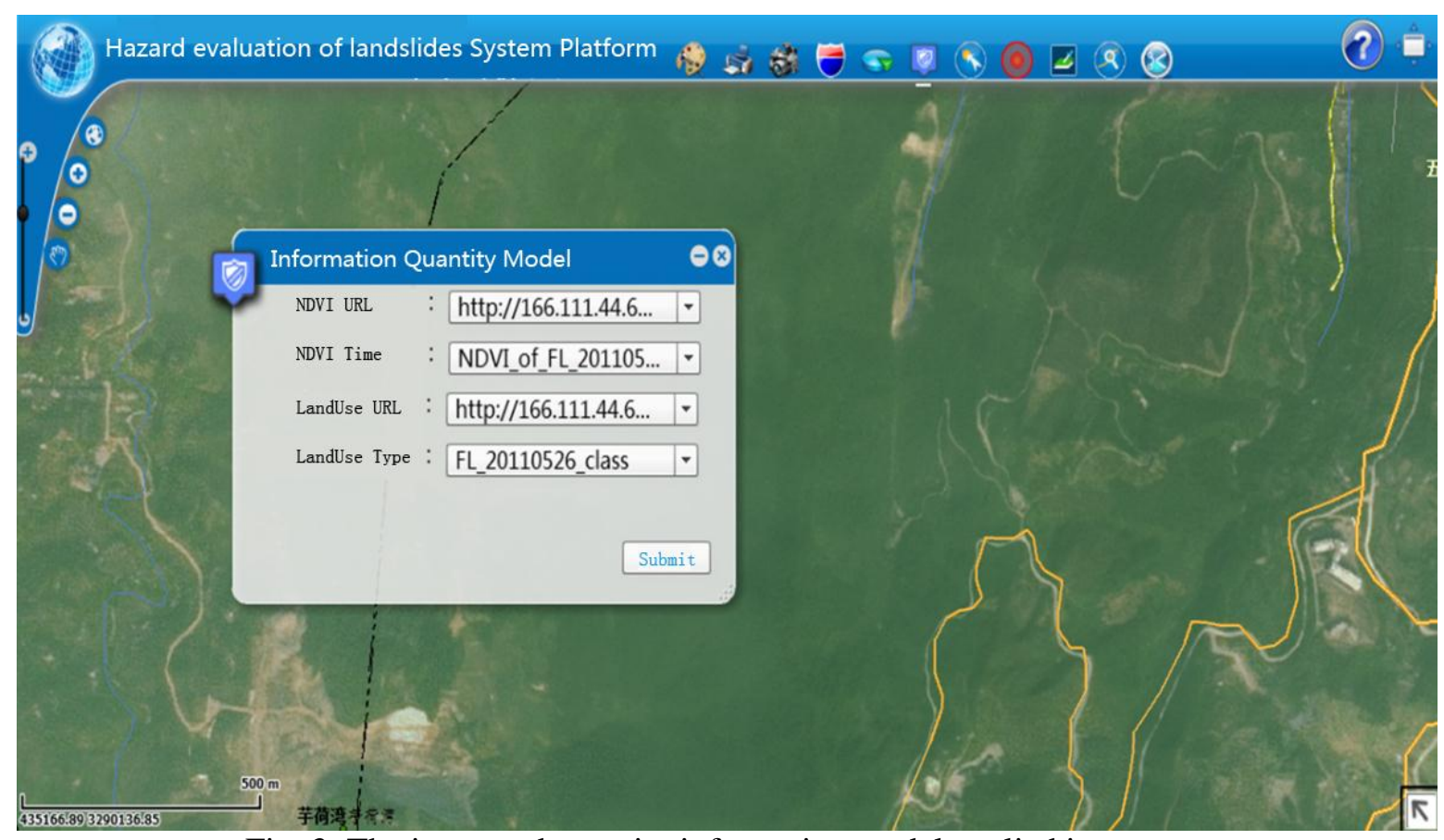

Fig. 3: The improved quantity information model applied in system.

\section{Conclusion}

Combined with theoretical basis of information model, we analysed the advantages and disadvantages in the factors and weights on hazard evaluation of landslide, then we put forward an improved information quantity mode combing with four impacts of external factors, rainfall, water level changes, vegetation cover and landuse, to improve accuracy of hazard evaluation. Also the key method of system realizing which is based on ArcGIS software platform, with a process model, and published on the hazard evaluation system of landslide in Chongqing, which obtain a better result and automatic process in the experimental area.

\section{References}

[1] Lee S, Ryu J H, Won J S, et al. Determination and application of the weights for landslide susceptibility mapping using an artificial neural network[J]. Engineering Geology, 2004, 71:289-302.

[2] Yin KunLong. Landslide hazard prediction[M]. Wuhan: Press of China University of Geosciences, 2001: 19-22.

[3] Zhou WeiQiang. Gorges Reservoir Geological Hazard Warning Model study area and warning information system [D]. Beijing: Department of Civil Engineering, Tsinghua University. 2011.

[4] Li Jun, Zhou ChengHu. Appropriate Grid Size for Terrain Based Landslide Risk Assessment in Lantau Island,Hong Kong. Jornal of remote sensing[J]. 2003, 7(2):86-92.

[5] H. R. Pourghasemi, H. R. Moradi , M. Mohammdi. Landslide Hazard Zoning Using Bayesian Theory[J]. Journal of Science and Technology of Agriculture and Natural Resources, Water and Soil Science, 2013, 16(62): 109-120. 